\title{
Influência do nível competitivo e da posição tática sobre parâmetros de desempenho aeróbio de atletas profissionais de futebol do Brasil
}

CDD. 20.ed. 796.022

796.5

\author{
Bruno Manfredini BARONI* \\ Ricardo Bertotti PICCOLI** \\ Ernesto Cesar Pinto LEAL JUNIOR ${ }^{* * *}$ \\ *Universidade Federal \\ do Rio Grande do Sul. \\ ${ }^{* *}$ Universidade de Ca- \\ xias do Sul. \\ ${ }^{* * *}$ Universidade Nove \\ de Julho.
}

\section{Resumo}

Este estudo objetivou verificar a influência do nível competitivo e da posição tática sobre parâmetros relacionados ao desempenho aeróbio de atletas profissionais de futebol. Foram analisadas 453 avaliações ergoespirométricas de atletas profissionais de futebol (42 goleiros, 92 zagueiros, 61 laterais, 174 meiocampistas e 84 atacantes) que atuavam em quatro níveis competitivos: Campeonato Nacional Série $A ;$ Série $B$; Série $C$; e Campeonato Estadual. Não foram encontradas diferenças nos valores médios de consumo máximo de oxigênio $\left(\mathrm{VO}_{2 \max }\right)$ e segundo limiar ventilatório $\left(\mathrm{LV}_{2}\right)$ entre os niveis competitivos ( $\mathrm{p}$ $>0,05)$. Os goleiros apresentaram VO ${ }_{2 \max }$ e $\mathrm{LV}_{2}(56$ e $50 \mathrm{~mL} / \mathrm{kg} / \mathrm{min}$, respectivamente) significativamente inferiores aos zagueiros (59 e $52 \mathrm{~mL} / \mathrm{kg} \cdot \min ; p=0,002$ e $p=0,028$ ), laterais (60 e $53 \mathrm{~mL} / \mathrm{kg} \cdot \mathrm{min} ; \mathrm{p}<$ 0,001 e $p=0,004$ ), meio-campistas (59 e $52 \mathrm{~mL} / \mathrm{kg} \cdot \min ; p=0,002$ e $p=0,031$ ) e atacantes (59 e 52 $\mathrm{mL} / \mathrm{kg} \cdot \mathrm{min} ; \mathrm{p}=0,047$ e $p=0,036$ ). Portanto, os achados sugerem que: 1) o desempenho aeróbio não difere em função do o nível competitivo; e 2) os goleiros apresentam desempenho aeróbio inferior aos atletas de linha, os quais não apresentam diferenças entre si.

PalavRAS-Chave: Esporte; Ergoespirometria; Capacidade cardiorrespiratória.

\section{Introdução}

É amplamente difundido o conceito de que o desempenho dos atletas de futebol é altamente dependente de seu condicionamento físico ${ }^{1}$. Mesmo que as ações mais decisivas do jogo sejam executadas com predomínio do metabolismo anaeróbio (por exemplo, chutes, saltos e "sprints"), o futebol é um exercício de característica intermitente que exige dos atletas um elevado desempenho aeróbio ${ }^{2}$. Esse tipo de desempenho tem sido avaliado por meio dos valores de consumo máximo de oxigênio $\left(\mathrm{VO}_{2 \max }\right)$, limiar anaeróbio (LAn) e economia de corrida ${ }^{3}$, os quais podem ser incrementados a partir do treinamento específico dos atletas ${ }^{4}$.

Embora testes de campo possam ser empregados para se avaliar o desempenho aeróbio, as diferenças entre resultados mensurados em campo e em laboratório ${ }^{5}$ incentivam a utilização de testes ergoespirométricos. Esses testes são capazes de determinar com maior precisão o $\mathrm{VO}_{2 \max }$ e o segundo limiar ventilatório $\left(\mathrm{LV}_{2}\right)$, o qual coincide com o LAn, por meio da quantificação das trocas gasosas realizadas pelo avaliado durante o exercício ${ }^{1,6}$.

A maioria dos estudos acerca do desempenho aeróbio de atletas de futebol tem concentrado suas atenções sobre os valores de $\mathrm{VO}_{2 \max }$ e, em menor escala, de $\mathrm{LV}_{2}$. No entanto, informaçōes úteis para a prescrição e o monitoramento do treinamento desportivo têm sido negligenciadas, como, por exemplo, a intensidade de exercício em que o atleta atinge $\mathrm{o} \mathrm{LV}_{2}$ e o $\mathrm{VO}_{2 \max }$, um parâmetro que pode ser representado pela velocidade de corrida, pela frequência cardíaca ou pela sensação de esforço, entre outros.

Um número interessante de estudos tem se concentrado nas diferenças entre atletas que atuam nas 
diferentes posições táticas do futebol ${ }^{7-12}$. Os resultados demonstram que a menor solicitação do metabolismo aeróbio por parte dos goleiros conduz a menores valores de $\mathrm{VO}_{2 \max }$ e $\mathrm{LV}_{2}$ nesses atletas em relação aos jogadores de linha. No entanto, parece não haver um consenso em relação a possíveis diferenças entre laterais, zagueiros, meio-campistas e atacantes. Enquanto alguns estudos relatam inexistência de diferenças entre os atletas que atuam na linha ${ }^{8-10}$, superioridade em parâmetros aeróbios já foi encontrada em favor de meio-campistas ${ }^{7,11}$ e de atacantes ${ }^{12}$.

É possível identificar uma carência na literatura em relação à influência do nível competitivo em que os atletas atuam sobre os parâmetros de desempenho aeróbio. Evidências sugerem que atletas da primeira divisão apresentam valores de $\mathrm{VO}_{2 \max }$ superiores aos da segunda divisão em um país europeu ${ }^{10}$. Porém, existem marcantes diferenças na rotina de treinamento e no calendário de competiçōes a que os atletas são submetidos no Brasil e na Europa, tanto na divisão de elite quanto nas divisōes inferiores. Assim, uma vez que não foram encontrados estudos acerca da influência do nível competitivo sobre parâmetros aeróbios envolvendo atletas que atuam

\section{Método}

O presente trabalho caracteriza-se como um estudo de caráter transversal, onde foram analisadas 453 avaliações ergoespirométricas de atletas profissionais de futebol, realizadas entre os anos de 2003 e 2007, no Instituto de Medicina do Esporte e Ciências Aplicadas ao Movimento Humano da Universidade de Caxias do Sul (IME-UCS).

\section{Amostra}

Foram consideradas para análise 42 avaliações de goleiros e 411 avaliações de jogadores de linha, sendo 61 avaliaçóes de laterais, 92 de zagueiros, 174 de meio-campistas e 84 de atacantes. Todos os atletas integravam uma das cinco equipes profissionais participantes do estudo: uma equipe participante do Campeonato Brasileiro Série A $(\mathrm{n}=124)$, uma da Série $B(n=136)$, uma da Série $C(n=107)$ e duas equipes participantes apenas da primeira divisão do Campeonato Estadual do Rio Grande do Sul ( $\mathrm{n}=86)$.

Como critérios de inclusão, foram respeitados os seguintes pontos: 1) atletas profissionais de futebol, devidamente federados e que fizessem parte do grupo no Brasil, não se sabe se os resultados reportados por ARNASON et al. ${ }^{10}$ se aplicam à realidade brasileira.

Acerca dos fatos apresentados, o presente estudo compilou dados referentes às principais variáveis obtidas na avaliação ergoespirométrica de uma amostra numericamente expressiva de atletas profissionais que atuam no futebol brasileiro e comparou os valores obtidos pelos atletas que: 1) atuam em diferentes níveis competitivos, com o objetivo de verificar se o desempenho aeróbio pode ser apontado como um fator determinante do nível competitivo em que o atleta atua; e 2) atuam em diferentes posições táticas, com o objetivo de verificar a existência de diferenças no desempenho aeróbio de atletas que atuam como goleiros, laterais, zagueiros, meio-campistas e atacantes. As hipóteses do estudo eram de que: 1) atletas que atuassem em níveis competitivos mais elitizados apresentariam superioridade nos marcadores de desempenho aeróbio; e 2) atletas de linha apresentariam desempenho superior aos goleiros, ao mesmo tempo em que se esperava que a amostra numericamente expressiva permitisse a visualização de diferenças entre laterais, zagueiros, meio-campistas e atacantes.

de atletas das equipes participantes do estudo; 2) ausência de cardiopatias, diagnosticadas previamente por eletrocardiograma; 3) ausência de histórico de pneumopatias nos dois meses antecedentes aos testes; 4) ausência de lesōes osteomioarticulares que pudessem prejudicar seu desempenho nas avaliações ergoespirométricas; 5) adequada realização do teste ergoespirométrico, finalizando o mesmo devido à exaustão promovida pelo exercício e não por motivo outro qualquer; e 6 ) autorização da utilização dos dados pelos autores mediante assinatura de um Termo de Consentimento Livre e Esclarecido. Todas as avaliações de atletas que não obedeceram aos requisitos supracitados foram automaticamente excluídas do estudo.

\section{Procedimentos}

Todas as avaliaçōes ergoespirométricas foram conduzidas por uma equipe composta por profissionais de medicina, enfermagem e educação física. O mesmo médico com especialização em medicina esportiva conduziu todos os testes, os quais ocorreram em um ambiente padronizado, 
bem iluminado e com temperatura controlada em $24^{\circ} \mathrm{C}$. Os testes foram realizados de acordo com a preferência das comissóes técnicas de cada clube em relação ao período do ciclo de treinamento das temporadas esportivas.

Para a realização dos testes, foi utilizada uma esteira ergométrica modelo Super ATL (Inbrasport), um eletrocardiógrafo digital Elite (Micromed), um analisador de gases modelo $\mathrm{VO}_{2000}$ (MedGraphics) acoplado a um microcomputador equipado com o "software" específico para tal finalidade (Elite, Micromed), além de clipe nasal esterilizado, eletrodos descartáveis, lâminas de barbear descartáveis, lixas descartáveis e álcool 70\%.

O protocolo adotado caracteriza-se como um teste de esforço progressivo, com inclinação constante de $1^{\circ}$ e aumento gradativo da velocidade da esteira ergométrica. Após um período de três minutos de adaptação com caminhada a $4,0 \mathrm{~km} / \mathrm{h}$, o protocolo de avaliação era iniciado com velocidade de 6,0 $\mathrm{km} / \mathrm{h}$. Após o primeiro minuto de teste, a velocidade era incrementada em $2,0 \mathrm{~km} / \mathrm{h}$ (passando então para $8,0 \mathrm{~km} / \mathrm{h}$ ). A partir deste ponto, era realizado um incremento constante de $1,0 \mathrm{~km} / \mathrm{h}$ a cada minuto do teste, sendo que o término do teste era determinado pela exaustão do atleta, que sinalizava ao avaliador a incapacidade em prosseguir ${ }^{13}$. Em seguida, era realizado um período de recuperação a uma velocidade de $4,0 \mathrm{~km} / \mathrm{h}$, mantido pelo tempo necessário até que se atingisse a estabilização da frequência cardíaca e pressão arterial do atleta.

Uma tabela contendo a escala de BORG ${ }^{14}$ foi fixada imediatamente a frente da esteira e facilmente à vista do avaliado. Essa escala numérica engloba classificações de seis a 20 e visa estimar a percepção de esforço do indivíduo, indo da classificação "muito fácil" até a classificação "exaustivo". A análise da percepção de esforço foi realizada minuto-a-minuto durante o teste ergoespirométrico, quando o médico solicitava que o avaliado indicasse por meio de sinais previamente combinados qual o nível de esforço em que se encontrava naquele momento do teste.

A análise dos dados metabólicos obtidos em cada avaliação foi interpretada por um mesmo médico do esporte com experiência na área. $\mathrm{OVO}_{2 \max }$ foi definido como a intensidade de exercício na qual um platô da curva de $\mathrm{VO}_{2}$ foi observada. Quando um platô não pôde ser detectado na curva de $\mathrm{VO}_{2}$, foi utilizado o maior valor de $\mathrm{VO}_{2}$ detectado nos momentos finais de teste ${ }^{15-16}$. $\mathrm{O} \mathrm{LV}_{2}$ foi identificado mediante análise realizada pela plotagem dos dados metabólicos em gráficos, considerando o $\mathrm{LV}_{2}$ do indivíduo a carga mínima em que o equivalente ventilatório de oxigênio $\left(\mathrm{VE} / \mathrm{VO}_{2}\right)$ apresentou aumento concomitante com o incremento do equivalente ventilatório de dióxido de carbono $\left(\mathrm{VE} / \mathrm{VCO}_{2}\right)^{15,17-18}$.

\section{Análise de resultados}

As variáveis selecionadas para análise foram: 1) idade; 2) massa corporal; 3) estatura; 4) $\mathrm{VO}_{2 \max }$, em valores absolutos e normalizados pela massa corporal dos atletas; 5) velocidade de obtenção do $\mathrm{VO}_{2 \max }$; 6) frequência cardíaca (FC) no $\mathrm{VO}_{2 \text { max }}$; 7 ) percentual da frequência cardíaca máxima no $\mathrm{VO}_{2 \max }\left(\% \mathrm{FC}_{\text {max }}\right.$; calculada pela fórmula: $\mathrm{FC}_{\text {max }}=220$-idade); 8) $\mathrm{LV}_{2}$, em valores absolutos e normalizados pela massa corporal dos atletas; 9) velocidade de obtenção do $\mathrm{LV}_{2}$; 10) $\mathrm{FC} \mathrm{no} \mathrm{LV}_{2}$; 11) \% $\mathrm{FC}_{\text {max }}$ no $\mathrm{LV}_{2}$; percentual do $\mathrm{VO}_{2 \max }\left(\% \mathrm{VO}_{2 \max }\right)$ no $\mathrm{LV}_{2}$; e 12) escala subjetiva de esforço (Borg) no $\mathrm{LV}_{2}$.

Diante da esperada diferença existente entre goleiros e jogadores de linha, já verificada em estudos prévios do nosso grupo ${ }^{13}$, estas duas funçōes de jogo foram analisadas separadamente na comparação entre atletas de diferentes níveis competitivos (Série A, Série $\mathrm{B}$, Série Ce Estadual). As comparaçōes entre os grupos de atletas de cada nível competitivo foram realizadas por meio de uma One-Way Anova, seguida do "posthoc" de Bonferroni. As comparaçōes entre os atletas que atuam nas diferentes posiçôes táticas (goleiros, laterais, zagueiros, meio-campistas e atacantes) foram igualmente efetuadas por meio de uma One-Way Anova, seguida do "post-hoc" de Bonferroni.

A análise estatística foi desenvolvida no "software" SPSS 16.0, adotando-se um nível de significância de $5 \%(\mathrm{p}<0,05)$. Os valores obtidos são apresentados em média \pm desvio padrão.

\section{Resultados}

A análise estatística dos resultados obtidos pelos goleiros indicou efeito significativo do nível competitivo sobre as variáveis: massa corporal $(\mathrm{p}=0,003$;
$\mathrm{F}=5,631)$, estatura $(\mathrm{p}=0,005 ; \mathrm{F}=4,975)$ e velocidade no $\mathrm{VO}_{2 \max }(\mathrm{p}=0,031 ; \mathrm{F}=3,284)$ (TABELA 1). O teste "post-hoc" identificou que os goleiros 
da Série A apresentaram maior massa corporal que os goleiros da Série $C(p=0,007)$ e que os goleiros da Série B apresentaram superioridade em massa corporal e estatura em relação aos goleiros da Série $\mathrm{C}(\mathrm{p}=0,007$ e $\mathrm{p}=0,004$, respectivamente). Apesar do efeito significativo apontado pela análise estatística sobre a velocidade de obtenção do $\mathrm{VO}_{2 \max }$, não foram observadas diferenças significativas entre os grupos nesse ou em qualquer outro parâmetro de desempenho aeróbio.

TABELA 1 - Características antropométricas e parâmetros de desempenho aeróbio dos goleiros atuantes nos quatro níveis competitivos.

$\mathrm{VO}_{2 \max }=$ consumo máximo de oxigênio;

Veloc. $=$ velocidade;

$\mathrm{FC}=$ frequência cardíaca;

$\% \mathrm{FC}_{\text {max }}=$ percentual da frequência cardíaca máxima $\left(\mathrm{FC}_{\max }=\right.$ 220-idade);

$\mathrm{LV}_{2}=$ segundo limiar ventilatório;

Borg = escala de percepção de esforço;

* Significativamente maior que os atletas da Série C $(p<0,05)$.

\begin{tabular}{|c|c|c|c|c|c|}
\hline & Série A $(n=8)$ & Série $B(n=13)$ & Série $C(n=11)$ & Estadual $(n=10)$ & "p" \\
\hline Idade (anos) & $25,50 \pm 4,57$ & $25,50 \pm 4,57$ & $24,64 \pm 6,38$ & $23,50 \pm 4,72$ & 0,873 \\
\hline Massa corporal (kg) & $85,87 \pm 4,09 *$ & $85,00 \pm 6,00^{*}$ & $78,18 \pm 4,17$ & $83,70 \pm 3,77$ & 0,003 \\
\hline Estatura (m) & $1,86 \pm 0,03$ & $1,90 \pm 0,04^{*}$ & $1,84 \pm 0,03$ & $1,86 \pm 0,04$ & 0,005 \\
\hline $\mathrm{VO}_{2 \max }(\mathrm{L} / \mathrm{min})$ & $4,57 \pm 0,27$ & $4,75 \pm 0,29$ & $4,56 \pm 0,35$ & $4,64 \pm 0,36$ & 0,481 \\
\hline $\mathrm{VO}_{2 \max }(\mathrm{mL} / \mathrm{kg} \cdot \mathrm{min})$ & $53,32 \pm 3,75$ & $56,01 \pm 3,95$ & $58,52 \pm 5,40$ & $55,44 \pm 4,13$ & 0,097 \\
\hline Veloc. $\mathrm{VO}_{2 \max }(\mathrm{km} / \mathrm{h})$ & $16,25 \pm 0,46$ & $17,31 \pm 0,95$ & $17,27 \pm 0,90$ & $16,40 \pm 1,35$ & 0,031 \\
\hline $\mathrm{FC}$ no $\mathrm{VO}_{2 \max }(\mathrm{bpm})$ & $188,87 \pm 8,05$ & $187,85 \pm 8,31$ & $192,54 \pm 10,87$ & $191,30 \pm 9,85$ & 0,619 \\
\hline$\% \mathrm{FC}_{\max }$ no $\mathrm{VO}_{2 \max }$ & $97,14 \pm 4,59$ & $96,32 \pm 4,07$ & $98,54 \pm 4,18$ & $97,40 \pm 5,43$ & 0,699 \\
\hline $\mathrm{LV}_{2}(\mathrm{~L} / \mathrm{min})$ & $4,04 \pm 0,23$ & $4,18 \pm 0,24$ & $4,07 \pm 0,29$ & $4,07 \pm 0,34$ & 0,620 \\
\hline $\mathrm{LV}_{2}$ (mL/kg.min) & $47,18 \pm 3,41$ & $49,41 \pm 3,95$ & $52,15 \pm 3,99$ & $48,63 \pm 4,19$ & 0,055 \\
\hline Veloc. no $\mathrm{LV}_{2}(\mathrm{~km} / \mathrm{h})$ & $14,00 \pm 1,31$ & $14,69 \pm 1,11$ & $14,54 \pm 0,69$ & $14,10 \pm 1,10$ & 0,382 \\
\hline FC no $\mathrm{LV}_{2}$ (bpm) & $176,87 \pm 8,30$ & $174,38 \pm 9,39$ & $179,00 \pm 9,42$ & $178,70 \pm 7,35$ & 0,557 \\
\hline$\% \mathrm{FC}_{\max }$ no $\mathrm{LV}_{2}$ & $90,95 \pm 3,95$ & $89,42 \pm 4,78$ & $91,63 \pm 4,17$ & $90,99 \pm 4,44$ & 0,647 \\
\hline$\% \mathrm{VO}_{2 \max }$ no $\mathrm{LV}_{2}$ & $88,49 \pm 1,13$ & $88,23 \pm 3,79$ & $89,36 \pm 5,23$ & $87,69 \pm 3,21$ & 0,784 \\
\hline Borg no $\mathrm{LV}_{2}$ & $15,00 \pm 1,06$ & $14,54 \pm 1,20$ & $14,73 \pm 1,01$ & $14,00 \pm 0,94$ & 0,242 \\
\hline
\end{tabular}

Entre os atletas de linha, foi verificado efeito significativo do nível competitivo sobre as variáveis: massa corporal ( $\mathrm{p}=0,014 ; \mathrm{F}=3,559)$, estatura ( $\mathrm{p}$ $=0,041 ; \mathrm{F}=2,772)$ e velocidade no $\mathrm{VO}_{2 \max }(\mathrm{p}=$ $0,042 ; \mathrm{P}=2,763)$ (TABELA 2). O teste "poct-hoc" identificou a superioridade dos atletas da Série A em relação aos da Série B na variável massa corporal $(\mathrm{p}=0,010)$ e em relação aos atletas da Série $C$ na variável estatura $(\mathrm{p}=0,043)$. Novamente, não foram observadas diferenças significativas entre os grupos nos parâmetros de desempenho aeróbio.

A análise estatística apontou efeito significativo da posição desempenhada em campo sobre as variáveis: massa corporal $(\mathrm{p}<0,001 ; \mathrm{F}=40,637)$, estatura $(\mathrm{p}<0,001$; $\mathrm{F}=67,741), \mathrm{VO}_{2 \max }$ absoluto $(\mathrm{p}<0,001 ; \mathrm{F}=18,799)$; $\mathrm{VO}_{2 \max }$ relativo $(\mathrm{p}<0,001 ; \mathrm{F}=5,463)$, velocidade no $\mathrm{VO}_{2 \max }(\mathrm{p}<0,001 ; \mathrm{F}=6,878), \mathrm{LV}_{2}$ absoluto $(\mathrm{p}<0,001 ; \mathrm{F}$ $=16,670), \mathrm{LV}_{2}$ relativo $(\mathrm{p}=0,007 ; \mathrm{F}=3,552)$ e percentual da FC no $L_{2}$ ( $p=0,023 ; F=2,862$ ) (TABELA 3). De acordo com a análise realizada por meio do teste "posthoc", observamos as seguintes diferenças significativas:

1) Massa corporal: os goleiros apresentaram valores maiores que os laterais $(\mathrm{p}<0,001)$, zagueiros $(\mathrm{p}<$ $0,001)$, meio-campistas $(\mathrm{p}<0,001)$ e atacantes $(\mathrm{p}<$
$0,001)$; os zagueiros apresentaram valores superiores aos laterais $(\mathrm{p}<0,001)$, meio-campistas $(\mathrm{p}=0,000)$ e atacantes $(\mathrm{p}<0,001)$; e os atacantes se mostraram mais pesados que os laterais $(\mathrm{p}<0,001)$;

2) Estatura: os goleiros apresentaram valores maiores que os laterais $(\mathrm{p}<0,001)$, zagueiros $(\mathrm{p}=0,018)$, meio-campistas ( $\mathrm{p}<0,001)$ e atacantes $(\mathrm{p}<0,001)$; os zagueiros eram mais altos que os laterais $(\mathrm{p}<0,001)$, meio-campistas $(\mathrm{p}<0,001)$ e atacantes $(\mathrm{p}<0,001)$;

3) $\mathrm{VO}_{2 \max }:$ em valores absolutos (mensurados $\mathrm{em} \mathrm{L} / \mathrm{min}$ ), os goleiros e os zagueiros apresentaram superioridade em relação aos laterais $(\mathrm{p}=0,001 ; \mathrm{p}$ $<0,001)$, meio-campistas ( $\mathrm{p}=0,001 ; \mathrm{p}<0,001)$ e atacantes $(\mathrm{p}=0,012 ; \mathrm{p}<0,001)$; porém, em valores normalizados pela massa corporal (mensurados em $\mathrm{mL} / \mathrm{kg} . \mathrm{min}$ ), os goleiros apresentaram valores inferiores aos laterais $(\mathrm{p}<0,001)$, zagueiros $(\mathrm{p}=0,002)$, meio-campistas $(\mathrm{p}=0,002)$ e atacantes $(\mathrm{p}=0,047)$;

4) Velocidade no $\mathrm{VO}_{2 \max }$ : os goleiros atingiram o $\mathrm{VO}_{2 \max }$ em velocidades inferiores aos laterais $(\mathrm{p}<$ $0,001)$, zagueiros $(\mathrm{p}<0,001)$, meio-campistas $(\mathrm{p}=$ $0,001)$ e atacantes $(\mathrm{p}=0,007)$;

5) $\mathrm{LV}_{2}:$ em valores absolutos (mensurados em $\mathrm{L} / \mathrm{min}$ ), os goleiros e os zagueiros apresentaram 
superioridade em relação aos laterais $(\mathrm{p}=0,001 ; \mathrm{p}$ $<0,001)$, meio-campistas $(\mathrm{p}=0,001 ; \mathrm{p}<0,001)$ e atacantes $(\mathrm{p}=0,050 ; \mathrm{p}<0,001)$; porém, em valores normalizados pela massa corporal (mensurados em mL/kg.min), os goleiros apresentaram valores inferiores aos laterais $(\mathrm{p}=0,004)$, zagueiros $(\mathrm{p}=$ $0,028)$, meio-campistas $(\mathrm{p}=0,031)$ e atacantes $(\mathrm{p}$ $=0,046)$.

TABELA 2 - Características antropométricas e parâmetros de desempenho aeróbio dos jogadores de linha atuantes nos quatro níveis competitivos.

\begin{tabular}{|c|c|c|c|c|c|}
\hline & Série A $(n=116)$ & Série $B(n=123)$ & Série $C(n=96)$ & Estadual $(n=76)$ & "p" \\
\hline Idade (anos) & $23,72 \pm 4,37$ & $24,26 \pm 4,28$ & $23,92 \pm 4,67$ & $23,17 \pm 4,48$ & 0,403 \\
\hline Massa corporal (kg) & $76,34 \pm 5,69 \#$ & $73,62 \pm 7,18$ & $75,00 \pm 6,63$ & $75,63 \pm 7,13$ & 0,014 \\
\hline Estatura $(\mathrm{m})$ & $1,79 \pm 0,06^{*}$ & $1,78 \pm 0,06$ & $1,77 \pm 0,05$ & $1,78 \pm 0,06$ & 0,041 \\
\hline $\mathrm{VO}_{2 \max }(\mathrm{L} / \mathrm{min})$ & $4,50 \pm 0,50$ & $4,41 \pm 0,50$ & $4,44 \pm 0,40$ & $4,42 \pm 0,43$ & 0,434 \\
\hline $\mathrm{VO}_{2 \max }(\mathrm{mL} / \mathrm{kg} \cdot \min )$ & $58,97 \pm 4,94$ & $59,94 \pm 4,79$ & $59,32 \pm 4,67$ & $58,72 \pm 5,19$ & 0,294 \\
\hline Veloc. $\mathrm{VO}_{2 \max }(\mathrm{km} / \mathrm{h})$ & $17,50 \pm 0,97$ & $17,81 \pm 0,88$ & $17,51 \pm 0,91$ & $17,55 \pm 1,12$ & 0,042 \\
\hline $\mathrm{FC}$ no $\mathrm{VO}_{2 \max }(\mathrm{bpm})$ & $190,20 \pm 9,05$ & $189,21 \pm 9,33$ & $188,07 \pm 10,18$ & $189,79 \pm 10,05$ & 0,426 \\
\hline$\% \mathrm{FC}_{\max }$ no $\mathrm{VO}_{2 \max }$ & $96,93 \pm 4,59$ & $96,69 \pm 4,82$ & $95,92 \pm 4,84$ & $96,43 \pm 4,72$ & 0,465 \\
\hline $\mathrm{LV}_{2}(\mathrm{~L} / \mathrm{min})$ & $3,94 \pm 0,47$ & $3,86 \pm 0,48$ & $3,93 \pm 0,37$ & $3,90 \pm 0,45$ & 0,539 \\
\hline $\mathrm{LV}_{2}(\mathrm{~mL} / \mathrm{kg} \cdot \mathrm{min})$ & $51,63 \pm 5,16$ & $52,49 \pm 4,62$ & $52,55 \pm 4,03$ & $51,97 \pm 5,06$ & 0,415 \\
\hline Veloc. no $\mathrm{LV}_{2}(\mathrm{~km} / \mathrm{h})$ & $14,81 \pm 0,93$ & $14,96 \pm 0,92$ & $14,85 \pm 0,93$ & $14,84 \pm 1,01$ & 0,601 \\
\hline FC no $L_{2}(\mathrm{bpm})$ & $175,53 \pm 9,06$ & $174,08 \pm 8,63$ & $174,51 \pm 11,17$ & $174,58 \pm 8,93$ & 0,691 \\
\hline$\% \mathrm{FC}_{\max }$ no $\mathrm{LV}_{2}$ & $89,46 \pm 4,82$ & $88,96 \pm 4,53$ & $88,98 \pm 5,03$ & $88,70 \pm 4,17$ & 0,708 \\
\hline$\% \mathrm{VO}_{2 \max }$ no $\mathrm{LV}_{2}$ & $87,53 \pm 4,70$ & $87,62 \pm 4,17$ & $88,66 \pm 3,29$ & $88,50 \pm 3,31$ & 0,092 \\
\hline Borg no $\mathrm{LV}_{2}$ & $14,59 \pm 1,32$ & $14,67 \pm 1,48$ & $14,87 \pm 1,64$ & $14,42 \pm 1,28$ & 0,220 \\
\hline
\end{tabular}

$\mathrm{VO}_{2 \max }=$ consumo máximo de oxigênio; Veloc. = velocidade; $F C$ = frequência cardíaca;

$\% \mathrm{FC}_{\text {max }}=$ percentual da frequência cardíaca máxima $\left(\mathrm{FC}_{\max }=\right.$ 220-idade);

$\mathrm{LV}_{2}=$ segundo limiar ventilatório;

Borg = escala de percepção de esforço;

* Significativamente maior que os atletas da Série $C(p<0,05)$;

\# Significativamente maior que os atletas da Série $B(p<0,05)$.

TABELA 3 - Características antropométricas e parâmetros de desempenho aeróbio dos atletas atuantes nas diferentes posições táticas.

\begin{tabular}{|c|c|c|c|c|c|c|}
\hline & $\begin{array}{c}\text { Goleiros } \\
(\mathrm{n}=42)\end{array}$ & $\begin{array}{l}\text { Laterais } \\
(\mathrm{n}=61)\end{array}$ & $\begin{array}{l}\text { Zagueiros } \\
(\mathbf{n}=92)\end{array}$ & $\begin{array}{c}\text { Meias } \\
(\mathrm{n}=174)\end{array}$ & $\begin{array}{c}\text { Atacantes } \\
(\mathbf{n}=\mathbf{8 4})\end{array}$ & "p" \\
\hline Idade (anos) & $24,62 \pm 5,19$ & $22,54 \pm 3,22$ & $24,29 \pm 4,77$ & $23,71 \pm 4,44$ & $24,48 \pm 4,63$ & 0,059 \\
\hline Massa corporal (kg) & $83,07 \pm 5,47^{\mathrm{LMA}}$ & $71,65 \pm 4,93$ & $80,20 \pm 5,69^{\mathrm{LMA}}$ & $73,72 \pm 6,34$ & $74,77 \pm 6,61^{\mathrm{L}}$ & 0,000 \\
\hline Estatura $(\mathrm{m})$ & $1,87 \pm 0,04^{\mathrm{LZMA}}$ & $1,76 \pm 0,06$ & $1,84 \pm 0,04^{\mathrm{LMA}}$ & $1,77 \pm 0,05$ & $1,76 \pm 0,05$ & 0,000 \\
\hline $\mathrm{VO}_{2 \max }(\mathrm{L} / \mathrm{min})$ & $4,63 \pm 0,31^{\mathrm{LMA}}$ & $4,31 \pm 0,35$ & $4,76 \pm 0,48^{\mathrm{LMA}}$ & $4,35 \pm 0,41$ & $4,37 \pm 0,45$ & 0,000 \\
\hline $\mathrm{VO}_{2 \max }(\mathrm{mL} / \mathrm{kg} \cdot \min )$ & $56,01 \pm 4,58$ & $60,31 \pm 5,06^{\mathrm{G}}$ & $59,43 \pm 5,22^{\mathrm{G}}$ & $59,19 \pm 4,81^{\mathrm{G}}$ & $58,62 \pm 4,46^{\mathrm{G}}$ & 0,000 \\
\hline Veloc. $\mathrm{VO}_{2 \max }(\mathrm{km} / \mathrm{h})$ & $16,88 \pm 1,06$ & $17,86 \pm 1,02^{\mathrm{G}}$ & $17,65 \pm 0,98^{\mathrm{G}}$ & $17,53 \pm 0,95^{\mathrm{G}}$ & $17,51 \pm 0,89^{\mathrm{G}}$ & 0,000 \\
\hline $\mathrm{FC}$ no $\mathrm{VO}_{2 \max }(\mathrm{bpm})$ & $190,09 \pm 9,23$ & $188,68 \pm 10,07$ & $189,17 \pm 9,43$ & $189,20 \pm 9,77$ & $190,22 \pm 4,25$ & 0,860 \\
\hline$\% \mathrm{FC}_{\max }$ no $\mathrm{VO}_{2 \max }$ & $97,31 \pm 4,46$ & $95,56 \pm 4,98$ & $96,68 \pm 4,65$ & $96,41 \pm 4,91$ & $97,30 \pm 4,18$ & 0,194 \\
\hline $\mathrm{LV}_{2}(\mathrm{~L} / \mathrm{min})$ & $4,10 \pm 0,27^{\mathrm{LMA}}$ & $3,78 \pm 0,33$ & $4,18 \pm 0,48^{\mathrm{LMA}}$ & $3,81 \pm 0,41$ & $3,88 \pm 0,41$ & 0,000 \\
\hline $\mathrm{LV}_{2}(\mathrm{~mL} / \mathrm{kg} \cdot \mathrm{min})$ & $49,51 \pm 4,17$ & $52,90 \pm 4,73^{\mathrm{G}}$ & $52,25 \pm 5,14^{\mathrm{G}}$ & $51,91 \pm 4,71^{\mathrm{G}}$ & $52,04 \pm 4,30^{\mathrm{G}}$ & 0,007 \\
\hline Veloc. no $\mathrm{LV}_{2}(\mathrm{~km} / \mathrm{h})$ & $14,38 \pm 1,05$ & $15,01 \pm 1,02$ & $15,89 \pm 10,27$ & $14,85 \pm 0,94$ & $14,89 \pm 0,86$ & 0,382 \\
\hline FC no $\mathrm{LV}_{2}(\mathrm{bpm})$ & $177,09 \pm 8,66$ & $174,26 \pm 10,79$ & $175,14 \pm 8,50$ & $174,06 \pm 9,69$ & $175,76 \pm 8,82$ & 0,316 \\
\hline$\% \mathrm{FC}_{\max }$ no $\mathrm{LV}_{2}$ & $90,66 \pm 4,32$ & $88,24 \pm 5,19$ & $89,51 \pm 4,30$ & $88,68 \pm 4,69$ & $89,92 \pm 4,46$ & 0,023 \\
\hline$\% \mathrm{VO}_{2 \max }$ no $\mathrm{LV}_{2}$ & $88,44 \pm 3,70$ & $87,24 \pm 3,69$ & $87,93 \pm 4,22$ & $87,73 \pm 4,14$ & $88,80 \pm 3,70$ & 0,295 \\
\hline Borg no $\mathrm{LV}_{2}$ & $14,54 \pm 1,08$ & $14,93 \pm 1,45$ & $14,61 \pm 1,31$ & $14,54 \pm 1,41$ & $14,70 \pm 1,61$ & 0,426 \\
\hline
\end{tabular}

$\mathrm{VO}_{2 \max }=$ consumo máximo de oxigênio; Veloc. = velocidade; $F C=$ frequência cardíaca; $\% \mathrm{FC}_{\text {max }}=$ percentual da frequência cardíaca máxima $\left(\mathrm{FC}_{\max }=\right.$ 220-idade);

$\mathrm{LV}_{2}=$ segundo limiar ventilatório;

Borg = escala de percepção de esforço;

${ }^{G}$ Significativamente maior que os Goleiros $(p<0,05)$;

ᄂ Significativamente maior que os Laterais $(p<0,05)$;

z Significativamente maior que os Zagueiros $(p<0,05)$;

M Significativamente maior que os Meias ( $p$ $<0,05)$;

A Significativamente maior que os Atacantes $(p<0,05)$. 


\section{Discussão}

O presente estudo objetivou verificar a influência do nível competitivo e da posição tática de atletas profissionais do futebol brasileiro sobre parâmetros de desempenho aeróbio. Como principais achados, destacamos que: 1) não foram encontradas diferenças nos parâmetros de desempenho aeróbio entre os atletas que atuavam nos diferentes níveis competitivos avaliados (campeonato nacional séries A, B e C, e campeonato estadual); e 2) os goleiros apresentaram inferioridade em relação aos jogadores de linha (laterais, zagueiros, meio-campistas e atacantes), os quais não apresentaram diferenças entre si. Esses resultados refutam a primeira hipótese do estudo e suportam parcialmente a segunda hipótese do estudo.

O primeiro achado do estudo, ou seja, a ausência de diferenças entre os atletas profissionais que atuavam nos quatro níveis competitivos, discorda dos resultados reportados por ARNASSON et al. ${ }^{10}$ que, avaliando 226 atletas do futebol islandês, encontraram superioridade de $\mathrm{VO}_{2 \max }$ dos atletas da primeira (63,2 mL/kg.min) em relação à segunda divisão (61,7 mL/kg.min). A igualdade de valores observada no presente estudo, entretanto, denota que o condicionamento aeróbio parece não diferir entre os atletas que atuam nos diferentes níveis de competição no Brasil, talvez por possuírem uma rotina semelhante de treinamento sistematizado. Além disso, os resultados sugerem que não sejam os valores de desempenho aeróbio os fatores determinantes na definição do nível competitivo em que o atleta atua. Assim, fatores adicionais não avaliados pelo presente estudo poderiam ser os responsáveis pelo nível de atuação do atleta no Brasil, como, por exemplo, a força muscular, a capacidade anaeróbia, as valências técnico-táticas e/ou fatores de ordem psicológica.

Uma vez comprovada a similaridade entre os atletas que atuavam nos níveis competitivos avaliados, partiuse para a análise englobando o total da amostra para o segundo objetivo do estudo de caracterizar o perfil aeróbio de atletas de cada posição tática. Os valores de $\mathrm{VO}_{2 \max }$ dos atletas de linha (58-60 mL/kg.min) estão de acordo com STøLEN et al. ${ }^{2}$, que realizaram uma abrangente revisão sobre a fisiologia do futebol e, analisando mais de 30 estudos na área envolvendo atletas de diferentes níveis competitivos, concluíram que o $\mathrm{VO}_{2 \max }$ de atletas de futebol do sexo masculino varia entre 50 e $75 \mathrm{~mL} / \mathrm{kg}$.min. Para os goleiros, os autores reportam dados de 50 a $55 \mathrm{~mL} / \mathrm{kg} / \mathrm{min}$, muito próximos aos encontrados no presente estudo $(56 \mathrm{~mL} /$ kg.min). DíAZ et al. ${ }^{19}$ demonstraram a evolução da preparação física no futebol, apresentando resultados de 248 atletas profissionais de elite nas décadas de 70,80 e 90, onde os valores do $\mathrm{VO}_{2 \max }$ foram respectivamente de 52,54 e $57 \mathrm{~mL} / \mathrm{kg}$.min. Com relação ao $\mathrm{LV}_{2}$, os valores observados pelo presente estudo (49-53 mL/kg.min) apresentam-se superiores aos achados de AL-HazzAA et al. ${ }^{8}$, que avaliando jogadores da seleção saudita reportaram valores médios de $44 \mathrm{~mL} / \mathrm{kg}$.min. Também em relação ao $\mathrm{LV}_{2}$, já fora observado que este estágio é atingido entre $77 \%$ e $90 \%$ do $\mathrm{VO}_{2 \max }{ }^{2}$, valores que vão ao encontro dos resultados do presente estudo (87-89\%).

Os valores absolutos de $\mathrm{VO}_{2 \max } \mathrm{eLV}_{2}$ (apresentados em $\mathrm{L} / \mathrm{min}$ ) apontam para uma superioridade dos goleiros e zagueiros sobre as demais posiçôes táticas. A análise isolada destes parâmetros geraria a equivocada conclusão de que os atletas destas duas posições apresentam um condicionamento aeróbio superior aos demais. Entretanto, uma vez que o futebol é um esporte caracterizado pela corrida e outros movimentos realizados em campo, nos quais o atleta constantemente necessita suportar a própria massa corporal, a normalização dos valores de $\mathrm{VO}_{2 \max }$ e $\mathrm{LV}_{2}$ por esta variável antropométrica tornase fundamental para uma adequada interpretação dos resultados. Assim, uma vez que superioridade nas dimensôes corporais de goleiros e zagueiros em relação aos demais jogadores foi encontrada no presente estudo e é reforçada por trabalhos prévios $^{10,13,20}$, enfatiza-se a necessidade da análise dos valores de consumo de oxigênio ser individualizada de acordo com a massa corporal de cada atleta.

A partir da análise de valores normalizados (portanto, em $\mathrm{mL} / \mathrm{kg} . \mathrm{min}$ ), verifica-se que os goleiros apresentaram $\mathrm{VO}_{2 \max }$ e $\mathrm{LV}_{2}$ significativamente inferiores a todas as demais posições táticas de jogo, as quais não apresentaram diferenças significativas entre si. A diferença de $\mathrm{VO}_{2 \max }$ entre goleiros e jogadores de linha já foi relatada por trabalhos internacionais de DAVIS et al. ${ }^{7}$ e ARNASSON et al. ${ }^{10}$, assim como por BALIKIAN et al. ${ }^{9}$ e BARONI et al. ${ }^{13}$ em estudos com atletas brasileiros. Somado a estes achados, os resultados reportados por AL-HAZZAA et al. ${ }^{8}$ e BALIKIAN et al. ${ }^{9}$ concordam com os nossos em relação à ausência de diferenças nos valores de $\mathrm{VO}_{2 \max }$ e $\mathrm{LV}_{2}$ apresentados por laterais, zagueiros, meio-campistas e atacantes.

A inferioridade do desempenho aeróbio dos goleiros é corroborada pela análise da velocidade em que os atletas desta posição tática atingiram o $\mathrm{VO}_{2 \max }$, 
significativamente inferior aos jogadores de linha. Em outras palavras, os goleiros não suportaram intensidades de exercício (no caso, a velocidade de corrida) tão altas quanto os jogadores de linha. A provável justificativa encontra-se na diferenciada demanda física imposta sobre o goleiro durante o jogo. Enquanto atletas de linha percorrem 10 a $12 \mathrm{~km}$ por partida, a distância percorrida pelos goleiros é de aproximadamente $4 \mathrm{~km}^{2}$. Além disso, os goleiros apresentam uma função extremamente diferenciada dos demais atletas, na qual são priorizados movimentos de alta intensidade e curta duração (como nos saltos para defesas de chutes a gol e saídas da meta para interceptação de lançamentos e cruzamentos), dependendo predominantemente do sistema anaeróbio para o fornecimento de energia ${ }^{21}$. Em função dessa demanda diferenciada em campo, a rotina de treinamento dos goleiros difere substancialmente dos jogadores de linha em especificidade, volume e intensidade, justificando as diferenças no condicionamento aeróbio.

Não foram verificadas diferenças significativas na frequência cardíaca dos atletas das cinco posições táticas durante a obtenção do $\mathrm{LV}_{2}$ ou do $\mathrm{VO}_{2 \max }$. Com base nos resultados do presente estudo, pode-se afirmar que atletas de futebol (tanto goleiros quanto jogadores de linha) atingem o $\mathrm{LV}_{2}$ em intensidades de exercício correspondentes à 88-90\% da $\mathrm{FC}_{\text {max }}$, reforçando os valores entre 80 e $90 \%$ da $\mathrm{FC}_{\max }$ já reportados pela literatura ${ }^{1-2}$. Tendo em vista que a frequência cardíaca se constitui em um parâmetro largamente utilizado para fins de treinamento em equipes de futebol e outras modalidades esportivas, estes resultados possuem importância do ponto de vista prático para treinadores e preparadores físicos, pois sugerem que estes percentuais podem ser utilizados em um grupo de atletas sem a necessidade de individualização de acordo com as posições táticas.

A escala de Borg, desenvolvida na década de $70^{14}$, consiste em uma ferramenta bastante difundida e utilizada na prescrição e monitoramento da intensidade de exercício. Embora colocada em dúvida por alguns autores ${ }^{16}$, a escala de Borg parece apresentar satisfatória relação com indicadores fisiológicos como a concentração de lactato, a frequência cardíaca e o consumo de oxigênio ${ }^{22}$. Assim, os resultados apresentados no presente estudo permitem inferir que atletas profissionais de futebol atingem o $\mathrm{LV}_{2}$ em intensidades subjetivas de aproximadamente 14, quando utilizada esta ferramenta de mensuração. Logo, sugere-se que este parâmetro possa ser utilizado para fins de treinamento, especialmente na ausência de equipamentos de mensuração de frequência cardíaca.

A principal limitação do estudo se encontra no fato de as avaliações não terem sido realizadas em um mesmo período da temporada competitiva pelas equipes participantes. No entanto, salientase o rigor metodológico e o fato de uma mesma equipe de coleta de dados ter atuado durante as avaliaçôes como pontos positivos que aumentam a credibilidade dos nossos resultados. Além disso, tendo em vista que não foram encontrados estudos que apresentassem uma amostra de atletas profissionais de futebol tão expressiva do ponto de vista numérico, especialmente se tratando de estudos desenvolvidos no Brasil, acredita-se que os valores reportados pelo nosso estudo podem servir de parâmetro tanto para pesquisadores em estudos futuros quanto para treinadores e preparadores físicos em sua prática profissional.

Como conclusão, os resultados obtidos no presente estudo sugerem que: 1) os parâmetros representativos do condicionamento aeróbio não diferem entre atletas profissionais que atuam nos quatro níveis competitivos do futebol brasileiro avaliados; 2) os goleiros possuem condicionamento aeróbio inferior aos jogadores de linha, tanto pelos menores valores de consumo de oxigênio máximo $\left(\mathrm{VO}_{2 \max }\right)$ e de limiar anaeróbio $\left(\mathrm{LV}_{2}\right)$ quanto pela menor intensidade de exercício em que atingem a exaustão no teste incremental; e 3) não há diferenças entre os jogadores de linha (zagueiros, laterais, meio-campistas e atacantes) nas variáveis de desempenho aeróbio avaliadas. 


\begin{abstract}
Influence of competitive level and tactical position on parameters of aerobic performance in professional soccer athletes from Brazil
\end{abstract}

This study aimed to verify the influence of the competitive level and the tactical position on parameters related to aerobic performance of professional soccer players. 453 ergospirometric evaluations were analyzed from soccer professional athletes (42 goalkeepers, 92 full backs, 61 sideways, 174 midfielders and 84 forwards) that acted in four competitive levels: National Championship Series A; Series B; Series C; and State Championship. It was found no differences in mean values of maximal oxygen uptake $\left(\mathrm{VO}_{2 \max }\right)$ and second ventilatory threshold $\left(\mathrm{LV}_{2}\right)$ between the competitive levels $(p>0.05)$. The goalkeepers showed $\mathrm{VO}_{2 \max }$ and $\mathrm{LV}_{2}$ ( 56 and $50 \mathrm{~mL} / \mathrm{kg}$. $\mathrm{min}$, respectively) significant lower than full backs ( 59 and $52 \mathrm{~mL} / \mathrm{kg}$. $\mathrm{min} ; \mathrm{p}=0.002$ e $p=$ 0.028 ), sideways ( 60 and $53 \mathrm{~mL} / \mathrm{kg} \cdot \mathrm{min} ; \mathrm{p}=0.000$ e $p=0.004$ ), midfields ( 59 and $52 \mathrm{~mL} / \mathrm{kg} \cdot \mathrm{min} ; p=0.002$ e $p=0.031$ ) and forwards ( 59 and $52 \mathrm{~mL} / \mathrm{kg} \cdot \mathrm{min} ; p=0.047$ e $p=0.036$ ). Therefore, the findings suggest that: 1) the aerobic performance does not differ according the competitive level; and 2) the goalkeepers have aerobic performance inferior to out-field players, which do not show differences between them.

KEY WORDS: Sport; Ergospirometry; Cardiorrespiratory capacity.

\title{
Referências
}

1. Helgerud J, Engen LC, Wisløff U, Hoff J. Aerobic endurance training improves soccer performance. Med Sci Sports Exerc. 2001;33:1925-31.

2. Stølen T, Chamari K, Castagna C, Wisløff U. Physiology of soccer. Sports Med. 2005;35:501-3.

3. Chamari K, Moussa-Chamari I, Boussaidi L, Hachana Y, Kaouech F, Wisløff U. Appropriate interpretation of aerobic capacity: allometric scaling in adult and young soccer players. Br J Sports Med. 2005;39:97-101.

4. Hoff J, Wisløff U, Engen LC, Kemi OJ, Helgerud J. Soccer specific aerobic endurance training. Br J Sports Med. 2002;36:218-21.

5. Chamari K, Hachana Y, Ahmed YB, et al. Field and laboratory testing in young elite soccer players. Br J Sports Med. 2004;38:191-6.

6. Fletcher GF, Balady GJ, Amsterdam EA, et al. Exercise standards for testing and training: a statement for healthcare professionals from the American Heart Association. Circulation. 2001;104:1694-740.

7. Davis JA, Brewer J, Atkin D. Pre-season physiological characteristics of English first and second division soccer players. J Sports Sci. 1992;10:541-7.

8. Al-Hazzaa HM, Almuzaini KS, Al-Refaee SA, et al. Aerobic and anaerobic power characteristics of Saudi elite soccer players. J Sports Med Phys Fitness. 2001;41:54-61.

9. Balikian P, Lourenção A, Ribeiro LFP, Festuccia WTL, Neiva CM. Maximal oxygen uptake and anaerobic threshold in professional soccer players: comparison between different positions. Rev Bras Med Esporte. 2002;8:32-6.

10. Arnason A, Sigurdsson SB, Gudmundsson A, Holme I, Engebretsen L, Bahr R. Physical fitness, injuries, and team performance in soccer. Med Sci Sports Exerc. 2004;36:278-85.

11. McIntyre MC, Hall M. Physiological profile in relation to playing position of elite college Gaelic footballers. Br J Sports Med. 2005;39:264-6.

12. Gil SM, Gil J, Ruiz F, Irazusta A, Irazusta J. Physiological and anthropometric characteristics of young soccer players according to their playing position: relevance for the selection process. J Strength Cond Res. 2007;21:438-45.

13. Baroni BM, Couto W, Leal Junior ECP. Descriptive-comparative study of aerobic performance parameters between soccer and futsal athletes. Rev Bras Cineantropom Desempenho Hum. 2011;13:170-6.

14. Borg GA Perceived exertion. Exerc Sport Sci Rev. 1974;2:131-53.

15. Dekerle J, Baron B, Dupont L, Vanvelcenaher J, Pelayo P. Maximal lactate steady state, respiratory compensation threshold and critical power. Eur J Appl Physiol. 2003;89:281-8.

16. Midgley AW, McNaughton LR, Polman R, Marchant D. Criteria for determination of maximal oxygen uptake. Sports Med. 2007;37:1019-28. 
17. Wasserman K, McIlroy MB. Detecting the threshold of anaerobic metabolism in cardiac patients during exercise. Am J Cardiol. 1964;14:844-52.

18. McLellan TM. Ventilatory and plasma lactate response with different exercise protocols: a comparison of methods. Int J Sports Med. 1985;6:30-5.

19. Díaz FJ, Montaño JG, Melchor MT, et al. Changes of physical and functional characteristics in soccer players. Rev Invest Clin. 2003;55:528-34.

20. Reilly T, Bangsbo J, Franks A. Anthropometric and physiological predispositions for elite soccer. J Sports Sci. 2000;18:669-83.

21. Cometti G, Maffiuletti NA, Pousson M, Chatard JC, Maffulli N. Isokinetic strength and anaerobic power of elite, subelite and amateur French soccer players. Int J Sports Med. 2001;22:45-51.

22. Irving BA, Rutkowski J, Brock DW, et al. Comparison of Borg- and OMNI-RPE as markers of the Blood Lactate response to exercise. Med Sci Sports Exerc. 2006;38:1348-52.

ENDEREÇO

Bruno Manfredini Baroni

Laboratório de Pesquisa do Exercício

Escola de Educação Física

Universidade Federal do Rio Grande do Sul

R. Felizardo, 750 - Jardim Botânico

90690-200 - Porto Alegre - RS - BRASIL

e-mail: bmbaroni@yahoo.com.br
Recebido para publicação: 09/04/2012

1a. Revisão: 17/09/2012

2a. Revisão: 24/11/2012

3a. Revisão: 21/12/2012

Aceito: $14 / 01 / 2013$ 